STRUCTURAL BIOLOGY COMMUNICATIONS

ISSN 2053-230X

Received 6 May 2020

Accepted 25 June 2020

Edited by N. Sträter, University of Leipzig, Germany

Keywords: plant terpenoids; camphor; oxidoreductases; Rossmann fold; NAD; expression medium; protein solubility.

PDB reference: borneol dehydrogenase, $6 \mathrm{~m} 5 \mathrm{n}$

Supporting information: this article has supporting information at journals.iucr.org/f

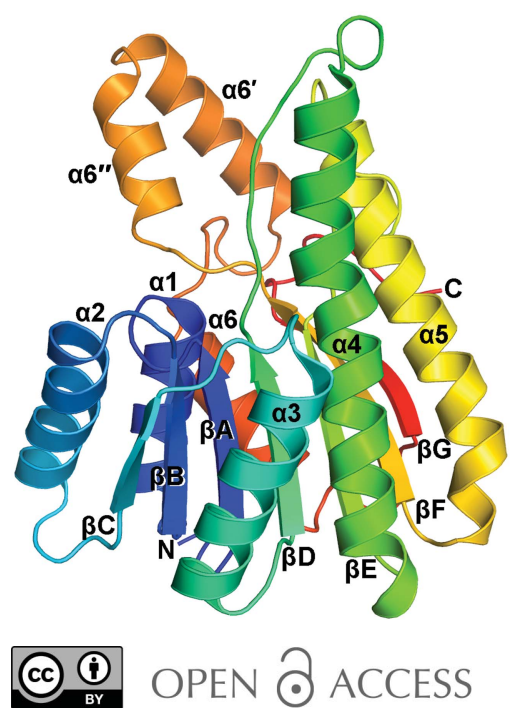

\section{Structural characterization of borneol dehydrogenase from Pseudomonas sp. TCU-HL1}

\author{
Aye Aye Khine, ${ }^{\mathrm{a}, \mathrm{b}, \mathrm{c}}$ Hao-Ping Chen, ${ }^{\mathrm{a}, \mathrm{b}, \mathrm{d}}$ Kai-Fa Huang ${ }^{\mathrm{e} *}$ and Tzu-Ping $\mathrm{Ko}^{\mathrm{e} *}$

 \\ Sciences, School of Medicine, Tzu Chi University, Hualien 97004, Taiwan, '⿳Department of Biochemistry, University of \\ Medicine 1, Yangon, Myanmar, dintegration Center of Traditional Chinese and Modern Medicine, Hualien Tzu Chi \\ Hospital, Hualien 97002, Taiwan, and Institute of Biological Chemistry, Academia Sinica, Taipei 11529, Taiwan. \\ *Correspondence e-mail: huangkf@gate.sinica.edu.tw, kotping@gate.sinica.edu.tw
}

During the microbial degradation of borneol, a bicyclic plant monoterpene, it is first converted into camphor by borneol dehydrogenase (BDH) and then enters a known camphor-degradation pathway. Previously, a recombinant Pseudomonas $\mathrm{BDH}$ was found in inclusion bodies when expressed in Escherichia coli. After refolding, it was still unstable and was difficult to concentrate. Here, the protein-expression conditions were improved by changing the medium from lysogeny broth to Terrific Broth, yielding a soluble form of the enzyme with higher activity. The protein was crystallized and its 3D structure was determined by X-ray diffraction. Like other known homologues such as quinuclidinone reductase, the protein forms a tetramer with subunits containing Rossmann folds. Structural comparison revealed major differences in the C-terminal helices and the associated loops. It is likely that these regions contain the determinants for substrate recognition.

\section{Introduction}

Borneol is one of the terpenoid alcohols that are naturally synthesized in plants and exists in two enantiomeric forms: $(+)$-borneol and (-)-borneol. (+)-Borneol is also a precursor in the biosynthetic pathway of natural (+)-camphor (Juteau et al., 2002). Borneol has commonly been used in traditional Chinese medicine for many years. It can relieve pain and serve as an analgesic and anesthetic agent (Hattori, 2000). Pharmacological effects such as anti-inflammation, neuroprotection and blood-brain barrier penetration have been reported (Liu et al., 2011; Zhong et al., 2014; Yin et al., 2017).

The degradation of borneol in microorganisms has previously been reported by our group (Tsang et al., 2016). Borneol dehydrogenase (BDH; accession No. WP_069084981) converts borneol into camphor in Pseudomonas sp. TCU-HL1 (Fig. 1). The camphor is then metabolized by this microorganism through a known camphor-degradation pathway (Ougham et al., 1983; Taylor \& Trudgill, 1986; Leisch et al., 2012; Iwaki et al., 2013). The gene encoding this enzyme, $b d h$, with locus tag TCL1_4180, was found in the camphordegradation gene cluster. Recombinant $\mathrm{BDH}$ was produced in the form of inclusion bodies in Escherichia coli BL21(DE3) cells. However, even after refolding, it was unstable and very easily precipitated. Consequently, various protein-induction methods have been tested in order to improve the solubility of this recombinant enzyme.

The $\mathrm{BDH}$ protein is homologous in primary sequence to a number of other oxidoreductases for which three-dimensional structures are available in the Protein Data Bank. The most similar is the 3-quinuclidinone reductase from Agrobacterium 
tumefaciens (AtQR; 36.4\% identity; PDB entries 3ak4 and 3 wds; Hou et al., 2014). The enzyme assembles into a tetramer of four identical subunits; each subunit contains a central parallel $\beta$-sheet with flanking $\alpha$-helices. Here, to provide a structural basis for future design and engineering to modify the substrate specificity of $\mathrm{BDH}$, we crystallized the protein and determined its 3D structure.

\section{Materials and methods}

\subsection{Macromolecule production}

The expression vector pbdhX, derived from pET-30 (Tsang et al., 2016), was used to transform E. coli BL21(DE3) cells for recombinant $\mathrm{BDH}$ production. The bacterial growth and induction conditions were the same as reported previously, except that Terrific Broth (TB) was used instead of lysogeny broth (LB). All purification steps were carried out at $4^{\circ} \mathrm{C}$. About $10 \mathrm{~g}$ of cells (wet weight) were resuspended in $50 \mathrm{ml}$ $10 \mathrm{~m} M$ sodium phosphate buffer $\mathrm{pH}$ 7.0, $1 \mathrm{~m} M$ phenylmethylsulfonyl fluoride (PMSF), $1 \mathrm{~m} M$ dithiothreitol (DTT) and were ruptured by sonication. The crude extracts were clarified by centrifugation at $25000 \mathrm{~g}$ for $15 \mathrm{~min}$. The supernatant was brought to $20 \%$ ammonium sulfate saturation, clarified by centrifugation and loaded onto a Phenyl Sepharose High Performance hydrophobic interaction column $(2.6 \times 70 \mathrm{~cm})$ pre-equilibrated with $400 \mathrm{ml}$ buffer $A$ (1 $M$ ammonium sulfate, $10 \mathrm{~m} M$ sodium phosphate $\mathrm{pH}$ 7). The proteins were eluted with buffer $A$ from 0 to $50 \mathrm{ml}$, with $100 \%$ buffer $A$ to $100 \%$ buffer $B(10 \mathrm{~m} M$ sodium phosphate $\mathrm{pH} 7)$ from 50 to $1050 \mathrm{ml}$ and with buffer $B$ from 1050 to $1250 \mathrm{ml}$. The BDH activity was determined using the procedure reported previously (Tsang et al., 2016), and fractions containing BDH activity were pooled. After overnight dialysis against buffer $B$ with $1 \mathrm{~m} M$ DTT, the protein solution was loaded onto a $\mathrm{Q}$ Sepharose Fast Flow anion-exchange column $(2.6 \times 50 \mathrm{~cm})$ and eluted using the method described above, in which buffer $A$ was replaced by buffer $B$ and the second buffer was buffer $B$ with $0.5 \mathrm{M} \mathrm{KCl}$. Macromolecule-production information is summarized in Table 1.

\subsection{Crystallization}

For crystallization experiments, the purified BDH was dialyzed and concentrated to $\sim 10 \mathrm{mg} \mathrm{ml}^{-1}$ in a new buffer consisting of $50 \mathrm{~m} M$ Tris- $\mathrm{HCl} \mathrm{pH} 8.0,5 \%$ glycerol. An initial crystallization screening of $\sim 800$ conditions was performed at the Protein Crystallization Facility, Institute of Biological Chemistry, Academia Sinica, Taipei, Taiwan. Single crystals were found in PEG-containing drops. After optimization,
Table 1

Macromolecule-production information.

\begin{tabular}{|c|c|}
\hline Source organism & Pseudomonas sp. TCU-HL1 \\
\hline DNA source & TCU-HL1 genomic DNA \\
\hline Forward primer $\dagger$ & $\begin{array}{l}5^{\prime} \text {-ATTTAGACATATGAAACTGCTAGAAGG } \\
\text { TAAAAGAATCATC- } 3^{\prime}\end{array}$ \\
\hline Reverse primer $\ddagger$ & $\begin{array}{l}5^{\prime}-\text { TAAGCGGATCCTAGCGTACAGCGTACA } \\
\text { GCGATCAGGCCAT-3' }\end{array}$ \\
\hline Cloning vector & pET-30a \\
\hline Expression vector & pET-30a \\
\hline Expression host & E. coli BL21(DE3) \\
\hline $\begin{array}{l}\text { Complete amino-acid sequence } \\
\text { of the construct produced }\end{array}$ & $\begin{array}{l}\text { MKLLEGKRI IVTGGAQGIGASVVRAYIAAG } \\
\text { ATVASMDMNDTLGQQVVSEAGKANPGCK } \\
\text { SRYYHCNIADRPEVEKAFATAAEDMGGL } \\
\text { DVMVNVAGVHRHSPPDAIAEELYDMLFR } \\
\text { VNVLGTINTNAVAYRLMKGQGIGNIINF } \\
\text { GSESGLTGEINNALYSATKAAVHTWTRN } \\
\text { VARQWGPDGIRINAVLPYMVTPMYVDFR } \\
\text { NALSSEDLAAHDAATKTDIPLGGKFGDA } \\
\text { DKDLAPVMVFLASDASHFMTGQMFPVDG } \\
\text { GLIAVR }\end{array}$ \\
\hline
\end{tabular}

$\dagger$ The NdeI site is underlined. $\ddagger$ The BamHI site is underlined.

Table 2

Crystallization.

\begin{tabular}{ll}
\hline Method & Sitting-drop vapor diffusion \\
Plate type & Intelli-Plate (Art Robbins Instruments) \\
Temperature $(\mathrm{K})$ & 293 \\
Protein concentration $\left(\mathrm{mg} \mathrm{ml}^{-1}\right)$ & 10 \\
$\begin{array}{l}\text { Buffer composition of protein } \\
\text { solution }\end{array}$ & $50 \mathrm{~m} M$ Tris- $\mathrm{HCl} \mathrm{pH} \mathrm{8.0,5 \%} \mathrm{glycerol}$ \\
Composition of reservoir solution & $30 \%(w / v)$ PEG 8000, $0.1 M$ sodium \\
& acetate $\mathrm{pH} 4.5,0.2 M$ lithium sulfate \\
Volume and ratio of drop & $5 \mu \mathrm{l}$ protein solution and $5 \mu \mathrm{l}$ reservoir \\
& solution $(1: 1)$ \\
Volume of reservoir $(\mu \mathrm{l})$ & 50
\end{tabular}

diffraction-quality crystals were obtained using a reservoir solution consisting of $30 \%(w / v)$ PEG $8000,0.1 M$ sodium acetate $\mathrm{pH} 4.5,0.2 \mathrm{M}$ lithium sulfate. The crystals were grown at $20^{\circ} \mathrm{C}$ by the sitting-drop vapor-diffusion method. Crystallization information is summarized in Table 2.

\subsection{Data collection and processing}

The crystals were first analyzed using the in-house X-ray facility at the Institute of Biological Chemistry, Academia Sinica. They belonged to the orthorhombic space group F222, with one BDH molecule in the asymmetric unit. High-resolution $\mathrm{X}$-ray diffraction data collection was carried out on the Taiwan Light Source (TLS) beamline BL15A1 at the National Synchrotron Radiation Research Center (NSRRC), Hsinchu, Taiwan. Prior to flash-cooling for data collection, the $\mathrm{BDH}$ crystals were soaked briefly in reservoir solution containing $10-15 \%(v / v)$ glycerol as a cryoprotectant. In an attempt to



Figure 1

Reaction catalyzed by BDH. 
Table 3

Data collection and processing.

Values in parentheses are for the outer shell.

\begin{tabular}{ll}
\hline Diffraction source & NSRRC beamline BL15A1 \\
Wavelength $(\AA)$ & 1.0000 \\
Temperature $(\mathrm{K})$ & 100 \\
Detector & Rayonix MX300HE CCD \\
Space group & $F 222$ \\
$a, b, c(\AA)$ & $71.63,96.51,142.56$ \\
$\alpha, \beta, \gamma\left({ }^{\circ}\right)$ & $90,90,90$ \\
Resolution range $(\AA)$ & $30.000-1.840(1.91-1.84)$ \\
Total No. of reflections & 107237 \\
No. of unique reflections & $21642(2120)$ \\
Completeness $(\%)$ & $100.0(100.0)$ \\
Multiplicity & $5.000(5.00)$ \\
Average $I / \sigma(I)$ & $11.54(2.03)$ \\
Average CC $1 / 2$ & $0.918(0.679)$ \\
$R_{\text {merge }}$ & $0.137(0.842)$ \\
$R_{\text {p.i.m. }}$ & $0.067(0.413)$ \\
Overall $B$ factor from Wilson plot $\left(\AA^{2}\right)$ & 20.51 \\
\hline
\end{tabular}

Table 4

Structure refinement.

Values in parentheses are for the outer shell.

\begin{tabular}{ll}
\hline Resolution range $(\AA)$ & $25.55-1.84(1.90-1.84)$ \\
Completeness $(\%)$ & $99.4(95.6)$ \\
No. of reflections, working set & $20451(1930)$ \\
No. of reflections, test set & $1076(101)$ \\
Final $R_{\text {cryst }}$ & $0.157(0.232)$ \\
Final $R_{\text {free }}$ & $0.182(0.274)$ \\
No. of non-H atoms & \\
$\quad$ Protein & 1927 \\
$\quad$ Ligand & 17 \\
$\quad$ Water & 285 \\
R.m.s. deviations & \\
$\quad$ Bond lengths $(\AA)$ & 0.005 \\
$\quad$ Angles $\left({ }^{\circ}\right)$ & 0.957 \\
Average $B$ factors $\left(\AA^{2}\right)$ & \\
$\quad$ Protein & 19.8 \\
$\quad$ Ligand & 44.4 \\
$\quad$ Water & 32.3 \\
Ramachandran plot & \\
$\quad$ Favored regions $(\%)$ & 97.3 \\
$\quad$ Additionally allowed $(\%)$ & 2.3 \\
Clashscore & 2.07 \\
MolProbity score & 1.19 \\
\hline
\end{tabular}

obtain complex crystals, $5 \mathrm{~m} M$ NAD was added to the reservoir solution. The diffraction intensities were integrated and scaled using HKL-2000 (Otwinowski \& Minor, 1997). Datacollection and processing statistics are summarized in Table 3.

\subsection{Structure solution and refinement}

The crystal structure was solved using MOLREP (Vagin \& Teplyakov, 2010) from CCP4 (Winn et al., 2011) with PDB entry 3ak4 as a search model and was refined to $1.84 \AA$ resolution using Phenix (Liebschner et al., 2019) and Coot (Emsley et al., 2010). Use of translation-libration-screw (TLS) parameters did not improve the $R$ values, and therefore all atoms were treated with isotropic temperature factors. MolProbity (Chen et al., 2010) was used for Ramachandran analysis. In addition to the NAD from PDB entry 3ak4, a model of camphor (CAM) was generated and both were placed into the active site of BDH using Phenix and Coot. Among the top $100 \mathrm{BDH}$-like structures in the PDB found by the DALI server (Holm, 2019), only a few contained bound substrates. These served as a guide for positioning the CAM model, which was subsequently improved by repeated manual adjustment and regularization according to the known structures and stereochemical restraints. In particular, the carbonyl group of CAM was oriented for the correct handedness of borneol upon hydride transfer. All figures showing 3D protein structures were produced using PyMOL (https://pymol.org/). Refinement statistics are summarized in Table 4.

\section{Results and discussion \\ 3.1. Expression of soluble BDH}

Most of the recombinant BDH was produced in a soluble form in crude extracts of $E$. coli using Terrific Broth as the growth medium. This was in contrast to the previous results using LB medium, in which the recombinant BDH was found in inclusion bodies and required refolding to solubilize it (Tsang et al., 2016). The specific activity of the soluble form of $\mathrm{BDH}$ turned out to be about 40-fold higher than that of the refolded enzyme. A homogeneous preparation of the enzyme was obtained after purification steps using hydrophobic and anion-exchange columns (Supplementary Fig. S1). The purified $\mathrm{BDH}$ was very stable and did not precipitate upon subsequent dialysis and concentration.

\subsection{Crystal structure of BDH}

The refined model of the orthorhombic $\mathrm{BDH}$ crystal contains an unbroken polypeptide chain from the N-terminal Lys2 to the C-terminal Arg260. As shown in Fig. 2(a), the protein features a seven-stranded antiparallel $\beta$-sheet flanked by three $\alpha$-helices on each side, making up a Rossmann-fold structure. The longer helices $\alpha 4$ and $\alpha 5$ on one side protrude over the $\mathrm{C}$-terminal ridge of the $\beta$-sheet, where the cofactor NAD is supposed to bind. On the other side, two additional helices $\alpha 6^{\prime \prime}$ and $\alpha 6^{\prime}$ are inserted between strand $\beta \mathrm{F}$ and helix $\alpha 6$. The space surrounded by these four $\alpha$-helices should participate in substrate binding and catalysis. However, soaking experiments failed to show electron density for NAD.

Although the $F 222$ crystal only contains one BDH protomer in the asymmetric unit, a similar tetramer to those of AtQR and other homologous oxidoreductases can be generated by crystallographic symmetry operations, as shown in Fig. 2(b). The protein-protein interface buries $3520 \AA^{2}(28 \%)$ of the total $12520 \AA^{2}$ surface area of each protomer. The tetramers of BDH and AtQR (PDB entry 3ak4) superimpose well, with an overall root-mean-square deviation (r.m.s.d.) of $1.69 \AA$ for 964 pairs of matched $\mathrm{C}^{\alpha}$ atoms (Supplementary Fig. S2). If individual protomers are compared, the r.m.s.d. ranges from 1.52 to $1.58 \AA$ for $239-242 \mathrm{C}^{\alpha}$ pairs. The regions that differ the most are located in helices $\alpha 6^{\prime \prime}, \alpha 6^{\prime}$ and $\alpha 6$ and the associated loops, as shown in Fig. 2(c).

\subsection{Substrate-binding mode}

The nicotinamide moiety of NAD from AtQR lies at the bottom of a cavity in $\mathrm{BDH}$, surrounded by loop $\beta \mathrm{D}-\alpha 4$, loop 
$\beta \mathrm{E}-\alpha 5$, loop $\beta \mathrm{F}-\alpha 6^{\prime \prime}$ and helix $\alpha 6^{\prime \prime}$ (Supplementary Fig. S3). While the NAD-binding site is largely conserved, the surrounding regions are variable, which should account for the binding to the preferred substrate. In this regard, three substrate-containing structures, PDB entries 1e6w (Powell et ;al., 2000), 3003 (Zhang et al., 2009) and 6ihh (Chen et al., 2019), were compared and a BDH substrate was modeled into the active site (Fig. 3). The plausible binding mode suggests

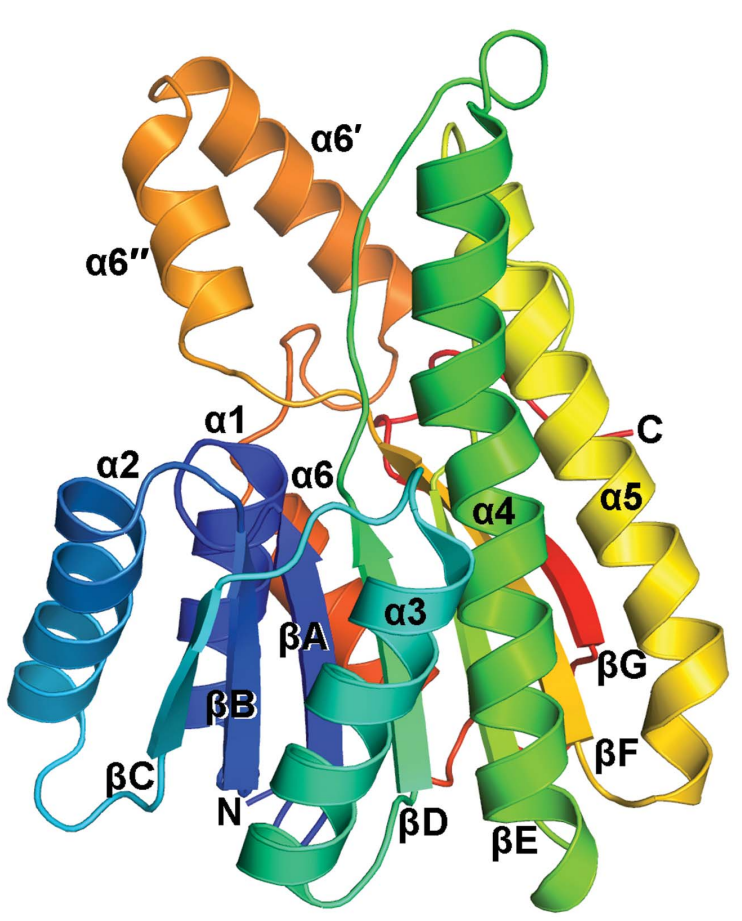

(a)

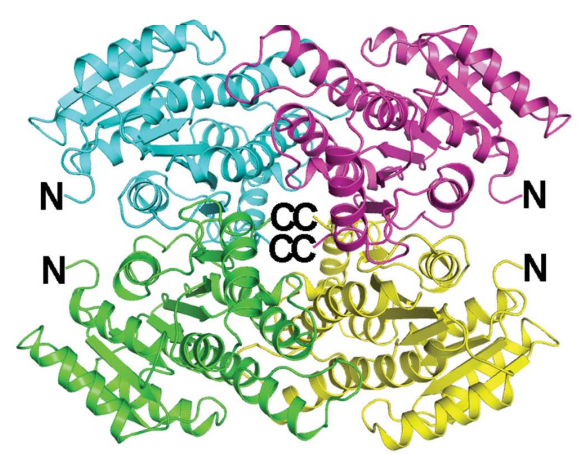

(b)

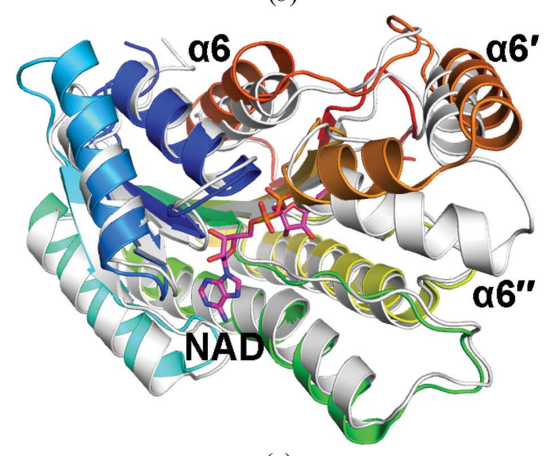

$(c)$

Figure 2

Overall structure of BDH. (a) The BDH monomer is shown as a ribbon diagram colored blue-cyan-green-yellow-red from the N-terminus to the C-terminus. The $\alpha$-helices and $\beta$-strands are denoted by numbers and letters. (b) The four monomers in a BDH tetramer are colored differently, with the $\mathrm{N}$ - and C-termini indicated. (c) The structure of BDH, presented as in (a), is compared with that of AtQR (PDB entry 3ak4), which is colored gray. Most of the Rossmann-fold part superimposes well, whereas helices $\alpha 6^{\prime \prime}, \alpha 6^{\prime}$ and $\alpha 6$ deviate significantly. The bound NAD in AtQR is shown as a stick model.



(a)



(b)

Figure 3

Substrate-binding region. (a) The monomers of BDH (with acetate; green) amd PDB entries 1e6w (rat HADH II/ABAD + estradiol; cyan), 3o03 (Streptococcus Ga5DH + gluconate; magenta) and 6ihh (Ralstonia cyclodiketone reductase + ethyl secodione; yellow) are superimposed and shown as wire models, along with the active-site-bound ligands, which are shown as thin sticks. The NAD from AtQR (gray) and the modeled camphor (blue) are shown as thick sticks. The BDH helices are labeled. $(b)$ The cofactor- and substrate-binding region of BDH is shown in a close-up view. Amino-acid side chains surrounding the substrate are shown as thin sticks. 
hydrogen bonding between the His96 and Tyr157 side chains and the borneol hydroxy group, as well as nonpolar contacts of the Phe233 side chain with the dimethyl group. However, the precise enzyme-substrate interactions have yet to be revealed by further investigations.

\section{Acknowledgements}

The authors are grateful to NSRRC for allocation of beam time and assistance with data collection.

\section{Funding information}

This work was supported by Tzu Chi University (grant No. TCMRC-P-108006) and the Academia Sinica (grant Nos. ASKPQ-109-TPP2 and AS-KPQ-109-ITAR-11).

\section{References}

Chen, V. B., Arendall, W. B., Headd, J. J., Keedy, D. A., Immormino, R. M., Kapral, G. J., Murray, L. W., Richardson, J. S. \& Richardson, D. C. (2010). Acta Cryst. D66, 12-21.

Chen, X., Zhang, H., Maria-Solano, M. A., Liu, W., Li, J., Feng, J., Liu, X., Osuna, S., Guo, R.-T., Wu, Q., Zhu, D. \& Ma, Y. (2019). Nat. Catal. 2, 931-941.

Emsley, P., Lohkamp, B., Scott, W. G. \& Cowtan, K. (2010). Acta Cryst. D66, 486-501.

Hattori, A. (2000). Yakushigaku Zasshi, 35, 49-54.

Holm, L. (2019). Bioinformatics, 35, 5326-5327.

Hou, F., Miyakawa, T., Kataoka, M., Takeshita, D., Kumashiro, S., Uzura, A., Urano, N., Nagata, K., Shimizu, S. \& Tanokura, M. (2014). Biochem. Biophys. Res. Commun. 446, 911-915.

Iwaki, H., Grosse, S., Bergeron, H., Leisch, H., Morley, K., Hasegawa, Y. \& Lau, P. C. K. (2013). Appl. Environ. Microbiol. 79, 3282-3293.
Juteau, F., Masotti, V., Bessière, J. M., Dherbomez, M. \& Viano, J. (2002). Fitoterapia, 73, 532-535.

Leisch, H., Shi, R., Grosse, S., Morley, K., Bergeron, H., Cygler, M., Iwaki, H., Hasegawa, Y. \& Lau, P. C. K. (2012). Appl. Environ. Microbiol. 78, 2200-2212.

Liebschner, D., Afonine, P. V., Baker, M. L., Bunkóczi, G., Chen, V. B., Croll, T. I., Hintze, B., Hung, L.-W., Jain, S., McCoy, A. J., Moriarty, N. W., Oeffner, R. D., Poon, B. K., Prisant, M. G., Read, R. J., Richardson, J. S., Richardson, D. C., Sammito, M. D., Sobolev, O. V., Stockwell, D. H., Terwilliger, T. C., Urzhumtsev, A. G., Videau, L. L., Williams, C. J. \& Adams, P. D. (2019). Acta Cryst. D75, 861-877.

Liu, R., Zhang, L., Lan, X., Li, L., Zhang, T.-T., Sun, J.-H. \& Du, G.-H. (2011). Neuroscience, 176, 408-419.

Otwinowski, Z. \& Minor, W. (1997). Methods Enzymol. 276, 307-326.

Ougham, H. J., Taylor, D. G. \& Trudgill, P. W. (1983). J. Bacteriol. 153, 140-152.

Powell, A. J., Read, J. A., Banfield, M. J., Gunn-Moore, F., Yan, S. D., Lustbader, J., Stern, A. R., Stern, D. M. \& Brady, R. L. (2000). J. Mol. Biol. 303, 311-327.

Taylor, D. G. \& Trudgill, P. W. (1986). J. Bacteriol. 165, 489-497.

Tsang, H.-L., Huang, J.-L., Lin, Y.-H., Huang, K.-F., Lu, P.-L., Lin, G.-H., Khine, A. A., Hu, A. \& Chen, H.-P. (2016). Appl. Environ. Microbiol. 82, 6378-6385.

Vagin, A. \& Teplyakov, A. (2010). Acta Cryst. D66, 22-25.

Winn, M. D., Ballard, C. C., Cowtan, K. D., Dodson, E. J., Emsley, P., Evans, P. R., Keegan, R. M., Krissinel, E. B., Leslie, A. G. W., McCoy, A., McNicholas, S. J., Murshudov, G. N., Pannu, N. S., Potterton, E. A., Powell, H. R., Read, R. J., Vagin, A. \& Wilson, K. S. (2011). Acta Cryst. D67, 235-242.

Yin, Y., Cao, L., Ge, H., Duanmu, W., Tan, L., Yuan, J., Tunan, C., Li, F., Hu, R., Gao, F. \& Feng, H. (2017). Neuroreport, 28, 506-513.

Zhang, Q., Peng, H., Gao, F., Liu, Y., Cheng, H., Thompson, J. \& Gao, G. F. (2009). Protein Sci. 18, 294-303.

Zhong, W., Cui, Y., Yu, Q., Xie, X., Liu, Y., Wei, M., Ci, X. \& Peng, L. (2014). Inflammation, 37, 1148-1157. 